\title{
Sensorless Unbalance Modelling and Estimation as an Ancillary Service for LV 4-Wire/3-Phase Power Converters
}

\author{
Andres Suárez-González, Pablo García, Ángel Navarro-Rodríguez, Geber Villa and Jose M. Cano \\ University of Oviedo. Dept.of Elec., Computer \& System Engineering \\ Gijón, 33204, Spain \\ e-mail: suarezandres@uniovi.es, garciafpablo@uniovi.es, navarroangel@uniovi.es, villageber@uniovi.es, jmcano@uniovi.es
}

\begin{abstract}
This paper describes a method to provide LV four-wire three-phase power converters with the capability of unbalance estimation as an ancillary service to the main role that they play in the distribution system (distributed generator, energy storage system, drive, etc). Typically, dedicated grid/load current sensors are needed to effectively comply with unbalance compensation tasks, increasing system cost and reducing reliability. This is due to the difficulties that arise in the extraction of the zero and negative voltage components from the voltages at the point of common coupling, such as the inadequate resolution of full-scaled voltage sensors and limited spectral separation. In this paper, the proposed method does not rely on additional sensors to those typically used in VSCs, and in any case, those sensors are limited to the point of connection of the power converter. Impedance estimation only using converter-side current sensors is implemented by adding a high frequency voltage excitation over the fundamental command. A new model approach is proposed for the real-time extraction of system impedance using a complex-valued compact form. Considering the voltage source at that frequency to be unique in the grid, it will be proved the impedance can be estimated and, thus, the resulting negative sequence current which is used for unbalance compensation. For the zero sequence, a special arrangement of the converter voltage sensors together with a repetitive controller is used.
\end{abstract}

\section{INTRODUCTION}

The adoption of distributed generation has increased in the last years due to both political and economic regulations [1], [2], emerging power electronics converters topologies which allow to improve network quality and reliability [3] and decrease in the costs for distributed generation. Power converters have been employed at the transmission network to improve power transmission capability, such as Static Synchronous Compensator (STATCOM) [4], [5], Static Synchronous Series Compensator (SSSC) [6] and Unified Power Flow Controller (UPFC) [4], [7]. Similar topologies have been later integrated at distribution levels, e.g. Active Power Filters (APF) [8]-[11],

\footnotetext{
The present work has been partially supported by the predoctoral grants program Severo Ochoa for the formation in research and university teaching of Principado de Asturias PCTI-FICYT under the grant ID BP14-135. This work is also supported in part by the Research, Technological Development and Innovation Program Oriented to the Society Challenges of the Spanish Ministry of Economy and Competitiveness under grants ENE2016-77919-R and DPI2017-89186-R and by the European Union through ERFD Structural Funds (FEDER).
}

allowing the increase of the distributed generation penetration and overcoming some problems related to the decentralized generation approach.

Among the problems related to distributed generation paradigm [12], [13], current and/or voltage unbalances or sags have recently received much attention [14]-[18]. There are several international standards which recommend maximum limits of the voltage unbalance: ANSI Standard C81.1 requires to keep it lower than 3\% [19], International Electrotechnical Commission (IEC) and the European Committee for Electrotechnical Standardization (CENELEC) recommends the limit of $2 \%$ [20], [21].

Unbalance compensation can be achieved by shunt [22], series [23], or series/shunt combination of the power converter [24], [25]. Among the three structures, series/shunt allows for an optimal [26], [27] sharing of the voltage/current compensation. However, it requires a dedicated converter for the compensation problem. Solely shunt converter compensation can lead to high compensation currents through the converters, reducing the active/reactive power supply capability, and thus some sharing mechanism among the APF is needed [15]. Sharing of unbalance can be done either by exchanging information among the microgrid converters or by an autonomous method which is implemented in the form of a droop control [15], [16], [18].

Measurement of the unbalanced components, i.e. negative and zero sequence voltages and currents, is a challenging task. Two options are currently used, each of those having an important drawback. When relaying on the voltages at the point of common coupling (PCC), they are greatly affected by the grid impedance value. In those cases where the converter is coupled to a strong grid, the reduced grid impedance makes the negative and zero sequence voltage values to be within the resolution limits of the voltage sensors [28]. Alternatively, current sensors can be used for the measurement of the negative and zero sequence currents. However, this needs for additional current sensors to be placed either at the load or at the grid side.

At this paper, it is proposed to overcome these limitations by a combination of novel techniques. First, a new mod- 
elling approach, able to determine the average and differential grid impedances using only converter-side variables, will be presented. Negative sequence will be measured from the converter current sensors and by adding an additional excitation high-frequency signal over the voltage command. This high-frequency signal will interact with the unbalance load, creating a high-frequency negative current that can be used for compensation. Zero sequence voltage will be measured by using a dedicated voltage sensor, fully scaled to this signal. This will allow to increase the resolution while, in conjunction with another two phase-neutral sensors, it will allow the synchronization of the power converter to operate normally. This contribution is based on the results presented in [29], also by the same authors. The present work extends in detail the mathematical modelling, adding new simulation and experimental results which corroborate the accuracy of the proposed modelling technique.

This paper is organized as follows. In section II, a novel modeling technique will be used to analytically highlight the coupling between the positive, negative and zero current sequences with non-balanced loads and voltage unbalances. The proposed analysis allows obtaining the expressions of the complex current vectors at the $\alpha \beta 0$ reference frame from the values of the voltages and loads at the $a b c$ reference frame using a compact representation. The method is not only valid for the analysis at the fundamental frequency, but also when a high frequency signal is injected by the converter. In section III, the proposed method for the unbalance estimation using a high frequency signal injection for the measurement of the unbalance from the converter-side current sensors is explained. The development includes the needed theoretical expressions as well as the experimental validation. In section IV, a compensation method based on the high-frequency modelling is presented. In section $\mathrm{V}$, the proposed zero sequence compensation strategy is explained. The method includes two main features: 1) the use of an special sensor arrangement in order to increase the resolution of the zero sequence voltage and 2) the use of a repetitive controller for the compensation of the zero sequence current.

\section{SYSTEM MODELING}

Modelling of the system shown in Fig. 1 can be done both in the stationary reference frame $(\alpha \beta 0)$ or in a synchronous reference frame $(d q 0)$. In this paper, the $\alpha \beta 0$ reference frame is used, since it leads to a simpler formulation in the presence of unbalances [30]. Considering $R L$ elements for describing the system impedances, the voltage drop over each of the elements in the natural abc reference frame is given by (1).

$$
\mathbf{v}_{a b c}=\mathbf{R}_{a b c} \mathbf{i}_{a b c}+\mathbf{L}_{a b c} \frac{d \mathbf{i}_{a b c}}{d t}
$$

Transformation from $a b c$ to $\alpha \beta 0$ is defined in (2a) for the currents and voltages and in (2b) for the impedances, being the A matrix transformation defined in (3).

$$
\mathbf{x}_{a b c}=\mathbf{A} \mathbf{x}_{\alpha \beta 0}
$$

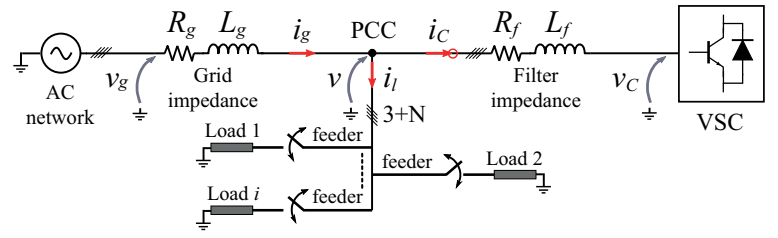

Fig. 1. Schematic for power converter and load connection to the point of common coupling. Loads can be both $3 \phi$ or $1 \phi$.

$$
\begin{gathered}
\mathbf{Z}_{\alpha \beta 0}=\mathbf{A}^{-1} \mathbf{Z}_{a b c} \mathbf{A} \\
\mathbf{A}=\sqrt{\frac{2}{3}}\left(\begin{array}{ccc}
1 & 0 & \frac{1}{\sqrt{2}} \\
-\frac{1}{2} & \frac{\sqrt{3}}{2} & \frac{1}{\sqrt{2}} \\
-\frac{1}{2} & -\frac{\sqrt{3}}{2} & \frac{1}{\sqrt{2}}
\end{array}\right)
\end{gathered}
$$

Moving from the $a b c$ to the $\alpha \beta 0$ reference frame has the advantage of using the more compact complex vector notation for the modelling, including the symmetrical component analysis which is widely used [31]. Under this transformation, the impedance matrix is expressed according to (2b) leading to (4).

$$
\mathbf{Z}_{\mathbf{a b c}}=\left(\begin{array}{ccc}
Z_{a} & 0 & 0 \\
0 & Z_{b} & 0 \\
0 & 0 & Z_{c}
\end{array}\right) \Rightarrow\left(\begin{array}{ccc}
Z_{\alpha \alpha} & Z_{\alpha \beta} & Z_{\alpha 0} \\
Z_{\beta \alpha} & Z_{\beta \beta} & Z_{\beta 0} \\
Z_{0 \alpha} & Z_{0 \beta} & Z_{00}
\end{array}\right)
$$

As shown, the impedance in the $\alpha \beta 0$ reference frame has a more complicated expression, with terms outside of the main diagonal. This will make the modelling more difficult and thus, it is desirable to find a mathematical approach for its simplification.

This paper proposes a new modelling approach, in which multiple reference frames in the $\alpha \beta 0$ domain are used. A graphical representation for the proposal is shown in Fig 2. Each of the impedances is represented in a reference frame aligned with the unbalance direction $\left(\theta_{e}^{i}\right.$ in Fig. 2). Using that approach, and assuming there is no cross-coupling between the phases, the impedance can be represented by (5). In the case cross-coupling is considered, it will be represented by nondiagonal terms [32]. However, it should be noted that crosscoupling impedances in low-voltage distribution networks have, generally, a negligible value compared to the phase impedances [33]. The main characteristic of this reference frame is the matrix being diagonal, thus noticeable easing the needed algebra for the modelling.

$$
\mathbf{Z}_{\alpha \beta \mathbf{0}_{i}}=\left(\begin{array}{ccc}
Z_{\alpha \alpha i} & 0 & 0 \\
0 & Z_{\beta \beta_{i}} & 0 \\
0 & 0 & Z_{00 i}
\end{array}\right)
$$

In (5), each load is represented by the $i$ subscript. For the case the load is balanced, $Z_{\alpha \alpha i}=Z_{\beta \beta_{i}}=Z_{00 i}$. Rotating the 


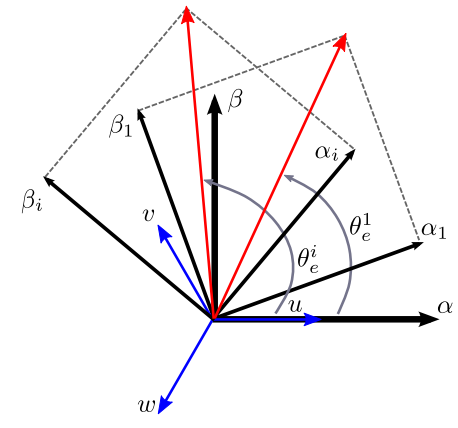

Fig. 2. Axis orientation for system loads $\left(\alpha_{i} \beta_{i}\right)$ and general stationary reference frame axis $(\alpha \beta)$.

impedance matrix to the $\alpha \beta 0$ reference frame and considering $n$ loads, leads to (6).

$$
\begin{aligned}
\mathbf{Z}_{\alpha \beta 0} & =\sum_{i=1}^{n}\left[\Sigma Z_{i}\left(\begin{array}{ccc}
1 & 0 & 0 \\
0 & 1 & 0 \\
0 & 0 & 1
\end{array}\right)\right. \\
& \left.+\left|\Delta Z_{i}\right|\left(\begin{array}{ccc}
\cos \theta_{e}^{i} & -\sin \theta_{e}^{i} & \sqrt{2} \cos \theta_{e}^{i} \\
-\sin \theta_{e}^{i} & -\cos \theta_{e}^{i} & \sqrt{2} \sin \theta_{e}^{i} \\
\sqrt{2} \cos \theta_{e}^{i} & \sqrt{2} \sin \theta_{e}^{i} & 0
\end{array}\right)\right]
\end{aligned}
$$

In (6), $\Sigma Z_{i}$ and $\Delta Z_{i}$ can be defined as (7) and (8) respectively.

$$
\begin{gathered}
\Sigma Z_{i}=\frac{Z_{\alpha \alpha i}+Z_{\beta \beta_{i}}}{2}=\frac{Z_{a}+Z_{b}+Z_{c}}{3} \\
\Delta Z_{i}=\frac{Z_{\alpha \alpha i}-Z_{\beta \beta_{i}}}{2}=\frac{Z_{a}+a \cdot Z_{b}+a^{2} \cdot Z_{c}}{3} \\
\theta_{e}^{i}=\tan ^{-1}\left(\Delta Z_{i}\right)
\end{gathered}
$$

$\theta_{e}^{i}$ is the impedance unbalance angle and $a=e^{-j 2 \pi / 3}$. For example, for single phase loads at phases $a, b, c, \theta_{e}^{i}$ equals $0,2 \pi / 3$ and $4 \pi / 3$ respectively. It is worth noting that for the case of single phase loads, in which the impedance at the two remaining phases are infinity, the expressions (7) and (8) will be also evaluated as infinity.

By substituting (6) in the voltage equation (1) expressed in the $\alpha \beta 0$ reference frame, and considering steady state conditions, the resulting current vector $\left(\mathbf{i}_{\alpha \beta}\right)$ is given by (10) and the homopolar component $\left(i_{0}\right)$ by (11).

$$
\begin{array}{r}
\mathbf{i}_{\alpha \beta}=V_{\alpha \beta}^{+} \sum_{i=1}^{n}\left[\Sigma Y_{i} e^{j \omega_{e} t}-\Delta Y_{i} e^{-j\left(\omega_{e} t+\theta_{e}^{i}\right)}\right] \\
+V_{\alpha \beta}^{-} \sum_{i=1}^{n}\left[\Sigma Y_{i} e^{-j \omega_{e} t}-\Delta Y_{i} e^{j\left(\omega_{e} t-\theta_{e}^{i}\right)}\right] \\
i_{0}=V_{0} \sum_{i=1}^{n}\left[\frac{1}{\Sigma Z_{i}} \sin \left(\omega_{e} t+\phi_{e}\right)\right] \\
+V_{\alpha \beta}^{+} \sum_{i=1}^{n}\left[\frac{\Delta Y_{i}^{2}}{\Sigma Y_{i}} \sqrt{2}\left(e^{j \omega_{e} t}-\frac{\Sigma Y_{i}}{\Delta Y_{i}} e^{j\left(\omega_{e}+\theta_{e}^{i}\right)}\right)\right] \\
+V_{\alpha \beta}^{-} \sum_{i=1}^{n}\left[\frac{\Delta Y_{i}^{2}}{\Sigma Y_{i}} \sqrt{2}\left(e^{-j \omega_{e} t}-\frac{\Sigma Y_{i}}{\Delta Y_{i}} e^{-j\left(\omega_{e}+\theta_{e}^{i}\right)}\right)\right]
\end{array}
$$

where $\Sigma Y_{i}=\frac{\Sigma Z_{i}}{\Sigma Z_{i}^{2}-\Delta Z_{i}^{2}}, \Delta Y_{i}=\frac{\Delta Z_{i}}{\Sigma Z_{i}^{2}-\Delta Z_{i}^{2}}, \omega_{e}$ is the grid frequency, $\phi_{e}=\tan ^{-1}\left(\mathbf{v}_{\alpha \beta}\right)$, and $V_{\alpha \beta}^{+}, V_{\alpha \beta}^{-}$and $V_{0}$ are the positive, negative and zero sequence magnitudes of the voltage vector.

The proposed model allows obtaining a compact expression in time domain for the $\alpha \beta 0$ terms of the current vector depending on the equivalent impedance as seen from a voltage source. This will be used later for isolating the different parameters in real time, having applications that goes from impedance estimation to unbalance compensation. In order to compare the proposed model with the traditional symmetrical components approach, (12) provides the expression for the positive, negative and zero components derived from equations (10) and (11).

$$
\begin{gathered}
\left(\begin{array}{c}
I_{\alpha \beta}^{+} \\
I_{\alpha \beta}^{-} \\
I_{0}
\end{array}\right)=\sum_{i=1}^{n}\left[\Sigma Y_{i}\left(\begin{array}{ccc}
1 & 0 & 0 \\
0 & 1 & 0 \\
0 & 0 & 1
\end{array}\right)-\Delta Y_{i} .\right. \\
0 \\
\left.\left.\begin{array}{ccc}
e^{j \theta_{e}^{i}} & 0 \\
e^{j \theta_{e}^{i}} & 0 & 0 \\
\sqrt{2}\left(e^{j \theta_{e}^{i}}-\frac{\Delta Y_{i}}{\Sigma Y_{i}}\right) & \sqrt{2}\left(e^{j \theta_{e}^{i}}-\frac{\Delta Y_{i}}{\Sigma Y_{i}}\right) & \frac{\Delta Y_{i}}{\Sigma Y_{i}}
\end{array}\right)\right]\left(\begin{array}{c}
V_{\alpha \beta}^{+} \\
V_{\alpha \beta}^{-} \\
V_{0}
\end{array}\right)
\end{gathered}
$$

Equations (10) to (12) build the proposed model by adding together all the admittances at the PCC using the superposition theorem. The equivalent admittance at the PCC, later used for the impedance identification, can be defined as (13).

$$
\begin{aligned}
& Y_{e q}^{\alpha \beta}=\sum_{i=1}^{n}\left[\frac { 1 } { \Sigma Z _ { i } ^ { 2 } - \Delta Z _ { i } ^ { 2 } } \left(\Sigma Z_{i}\left(\begin{array}{ll}
1 & 0 \\
0 & 1
\end{array}\right)+\right.\right. \\
& \left.\left.\left|\Delta Z_{i}\right|\left(\begin{array}{cc}
-\cos \theta_{i} & \sin \theta_{i} \\
\sin \theta_{i} & \cos \theta_{i}
\end{array}\right)\right)\right] \\
& =\frac{1}{\Sigma Z_{e q}^{2}-\Delta Z_{e q}^{2}}\left(\Sigma Z_{e q}\left(\begin{array}{cc}
1 & 0 \\
0 & 1
\end{array}\right)+\right. \\
& \left.\left|\Delta Z_{e q}\right|\left(\begin{array}{cc}
-\cos \theta_{e q} & \sin \theta_{e q} \\
\sin \theta_{e q} & \cos \theta_{e q}
\end{array}\right)\right)
\end{aligned}
$$

For the online estimation of the equivalent impedance, the equivalent admittance is used instead. This is to avoid any division by zero when the current is zero. The equivalent mean and differential admittances can be obtained using (14) and (15). Clearly, the two equations can be solved in real time with a really low computational burden, thus allowing for a simple identification method. Equivalent expressions for the impedance terms are also included in (16) and (17), respectively.

$$
\begin{aligned}
& \Sigma Y_{e q}^{\alpha \beta}=\frac{i_{\alpha \beta}^{+} \cdot v_{\alpha \beta}^{+}-i_{\alpha \beta}^{-} \cdot v_{\alpha \beta}^{-}}{\left(v_{\alpha \beta}^{+}\right)^{2}-\left(v_{\alpha \beta}^{-}\right)^{2}} \\
& \Delta Y_{e q}^{\alpha \beta}=\frac{i_{\alpha \beta}^{-} \cdot v_{\alpha \beta}^{+}-i_{\alpha \beta}^{+} \cdot v_{\alpha \beta}^{-}}{\left(v_{\alpha \beta}^{+}\right)^{2}-\left(v_{\alpha \beta}^{-}\right)^{2}} \\
& \Sigma Z_{e q}^{\alpha \beta}=\frac{v_{\alpha \beta}^{+} \cdot i_{\alpha \beta}^{+}-v_{\alpha \beta}^{-} \cdot i_{\alpha \beta}^{-}}{\left(i_{\alpha \beta}^{+}\right)^{2}-\left(i_{\alpha \beta}^{-}\right)^{2}} \\
& \Delta Z_{e q}^{\alpha \beta}=\frac{v_{\alpha \beta}^{-} \cdot i_{\alpha \beta}^{+}-v_{\alpha \beta}^{+} \cdot i_{\alpha \beta}^{-}}{\left(i_{\alpha \beta}^{+}\right)^{2}-\left(i_{\alpha \beta}^{-}\right)^{2}}
\end{aligned}
$$


TABLE I

SYSTEM PARAMETERS

\begin{tabular}{c|c}
\hline Converter parameters & Value \\
\hline Power $(\mathrm{kW})$ & 100 \\
DC Link Voltage (V) & 850 \\
Max Current (A) & 200 \\
Switching frequency $(\mathrm{kHz})$ & 10 \\
AC Filter & $L_{f}=7 \mathrm{mH}, R_{f}=0.33 \Omega$ \\
Grid impedance & $L_{g}=9 \mathrm{mH}, R_{g}=0.02 \Omega$ \\
DC bus capacitor $(\mu \mathrm{F})$ & 700 \\
\hline Loads & $R_{l}=18 \Omega$ \\
\hline Three phase balance load & $R_{l s}=18 \Omega$ \\
\hline Single phase loads & \\
\hline Control tuning & 100 \\
\hline Sampling control loop $T_{c}(\mu \mathrm{s})$ & $K_{p}=0.088, K_{i}=13.2$ \\
DC voltage loop & 10.0 \\
Current control loop & 333 \\
\hline High frequency parameters & 10 \\
\hline Carrier voltage $(\mathrm{V})$ & 10 \\
Carrier frequency $(\mathrm{Hz})$ & $K_{p}=5.341, K_{i}=2221.4$ \\
\hline Converter switching frequency $(\mathrm{kHz})$ & \\
Sampling frequency $(\mathrm{kHz})$ &
\end{tabular}

The proposed model has been validated by numerical simulation and experimental tests. For that, different stepchanges in the load impedance values $\left(z_{a}, z_{b}, z_{c}\right)$ have been applied and the predicted model values for the current and the impedances have been compared with respect to the simulation and the experimental results. Real setup is presented in Fig. 3 following the same topology as the schematic in Fig.1. A 4-Wire/3-Phase VSC of 30kVA has been used to perform the experiments with the system parameters depicted in Table I. The control system is implemented within a Texas Instruments Digital Signal Controller (F28335). The VSC was operated in STATCOM mode, where $d$-axis is devoted to maintaining the DC-Link at nominal voltage. A constant reference of $10 \mathrm{~A}$ is set in the q-axis. The reason behind the VSC being injecting current is to illustrate the effect of the load unbalance over the converter current. At initial time, load impedance is balanced and equal for the three phases $\left(Z_{a}=Z_{b}=Z_{c}=18 \Omega\right)$. As expected, neither negative nor homopolar component are observed.

Figs. 4, 5, 6 show the experimental results in the $\alpha \beta 0$ reference frame for the converter voltage; converter current and grid voltage at the PCC respectively, under different load unbalance conditions in each phase. At instant 0.1 s, a $18 \Omega$ load is connected in parallel to phase $a$, decreasing its nominal value by $50 \%$. Voltage distortion is present at PCC, where $14.4 \mathrm{~V}$ of negative sequence is measured, as it is shown in Fig. 4. The presence of the unbalance is also reflected in both Figs. 5 and 6 where 11.2A of homopolar current starts flowing through the system. Similarly, $1.2 \mathrm{~A}$ of negative sequence current is measured from the converter side and $5.2 \mathrm{~A}$, from the grid side. Same test is repeated in the other two phases (results from unbalance in phase $b$ and $c$ are shown in the middle and right columns), obtaining similar results in the voltage and current magnitudes.

Fig. 7 compares the impedance values according to (7), (8) and (9) and the ones obtained from the experimental

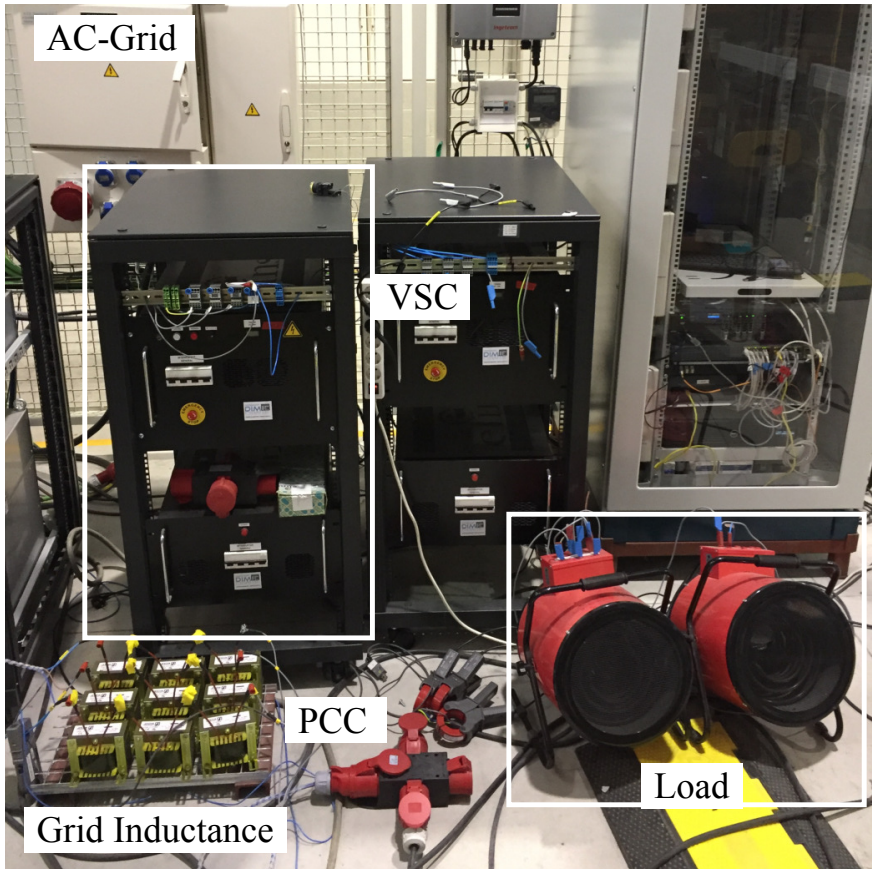

Fig. 3. Experimental setup according to the schematic shown in Fig.1. At the bottom left side the inductance bank used to emulate the impedance upstream from the PCC, where the STATCOM and local loads are connected.

measurements, at both grid and converter side, in order to support the proposed model and ease the explanation. The interesting aspect of the test lies on the comparison of the unbalance angle, $\theta_{e}^{i}$, which is rotating depending on the phase where the unbalance is located. In the first case, the impedance angle changes from $0^{\circ}$ to $-26.67^{\circ}$. The second case, where the unbalance takes place in phase $b, \theta_{i}^{e}$ changes from $0^{\circ}$ to $-148.3^{\circ}$. Notice that this result is $120^{\circ}$ delayed respect the previous unbalance in phase $a$, ideally should be $\theta_{b}=-26.67^{\circ}-120^{\circ}=-146.67^{\circ}$, The difference between the expected result and the real one is considered within the error tolerance. For the unbalance in phase $c$, the change is from $0^{\circ}$ to $93.33^{\circ}$, which corresponds to $\theta_{c}=120^{\circ}-26.67^{\circ}=$ $93.33^{\circ}$. The remaining variables depicted in Fig. 7 are not dependent on the phase unbalance but only on the magnitude of the unbalance. For this reason, $|\Sigma Z|$ decreases from $2.8 \Omega$ to $2.7 \Omega$ and $|\Delta Z|$ increases at instant 0.1 s from 0 to $0.15 \Omega$.

From (10), it is clear that the load unbalances will cause negative and zero sequence currents to appear, which potentially will create an unbalance in the grid supplied voltages. Compensation can be done by injecting a negative and zero sequence currents in opposition to the respective unbalanced currents [15]. There are several methods proposed in the literature to compensate for this, all of them requiring to separate (filter) the negative and zero sequences from the positive one and to cancel it out by using negative and zero sequence current controllers [15], [16]. For the measurement, extra current sensors for the load or the grid currents are required. The need for this extra sensor is demonstrated according to 


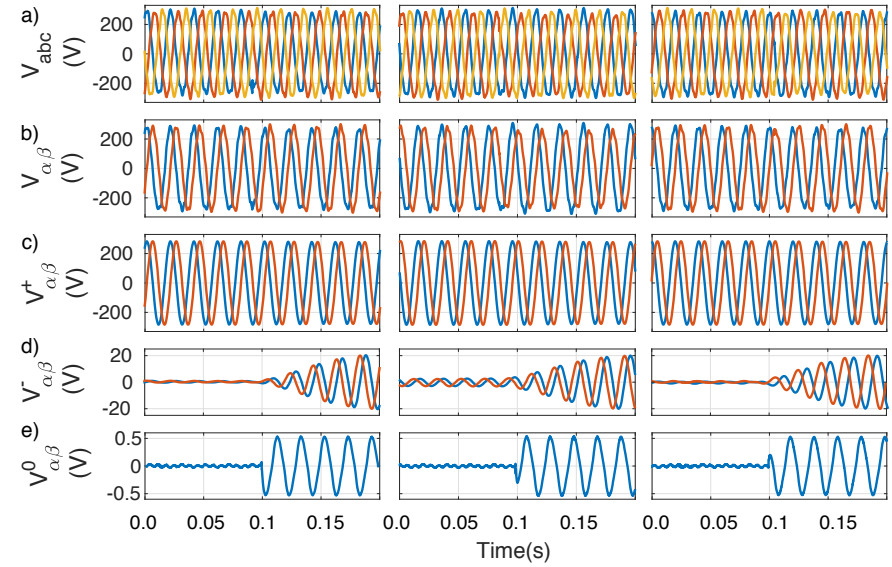

Fig. 4. Experimental results. Voltage measurements at the PCC, under three different unbalance conditions as consequence of a change in the load impedance. Unbalance is induced by connecting a parallel $18 \Omega$ load at $0.1 \mathrm{~s}$, to the $18 \Omega$ balanced load at the PCC. From left to the right: Unbalance induced in phase $a$, unbalance induced in phase $b$ and unbalance induced in phase $c$. From top to bottom: a) phase voltages, b) $\alpha, \beta$ components, c), d) and e) are the positive, negative and zero components of the voltage vector.

the results presented in Fig. 7, where the impedance observed from the converter side (green line) and the one observed from the grid side (red one) is represented. This clear inaccuracy in the results is due to the lack of information from the converter side currents. Impedances are calculated according to (16) and (17), resulting in (18) and (19).

$$
\begin{aligned}
& \Sigma Z_{g}^{\alpha \beta}=\frac{\left(v_{g_{\alpha \beta}}^{+}-v_{c_{\alpha \beta}}^{+}\right) \cdot i_{g_{\alpha \beta}}^{+}-\left(v_{g_{\alpha \beta}}^{-}-v_{c_{\alpha \beta}}^{-}\right) \cdot i_{g_{\alpha \beta}}^{-}}{\left(i_{g_{\alpha \beta}}^{+}\right)^{2}-\left(i_{g_{\alpha \beta}}^{-}\right)^{2}} \\
& \Delta Z_{g}^{\alpha \beta}=\frac{\left(v_{g_{\alpha \beta}}^{-}-v_{c_{\alpha \beta}}^{-}\right) \cdot i_{g_{\alpha \beta}}^{+}-\left(v_{g_{\alpha \beta}}^{+}-v_{c_{\alpha \beta}}^{+}\right) \cdot i_{g_{\alpha \beta}}^{-}}{\left(i_{g_{\alpha \beta}}^{+}\right)^{2}-\left(i_{g_{\alpha \beta}}^{-}\right)^{2}}
\end{aligned}
$$
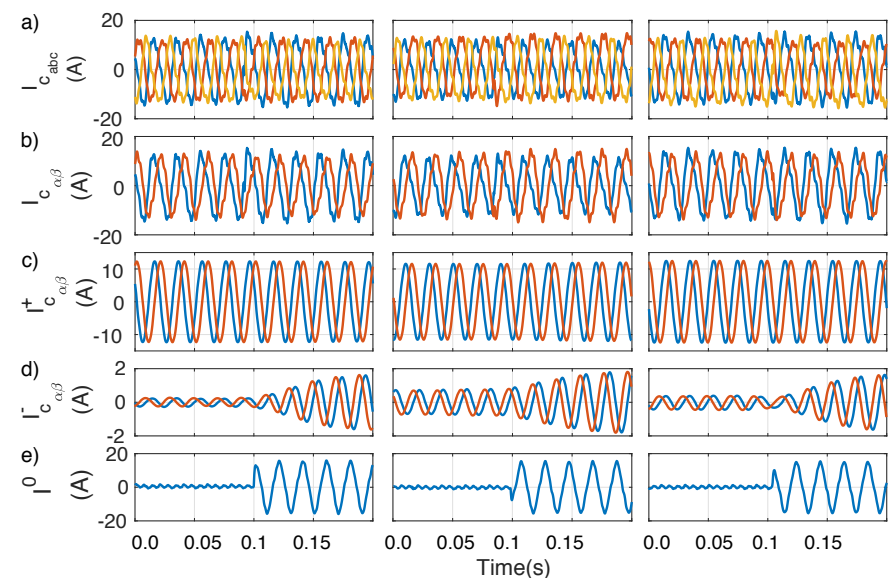

Fig. 5. Experimental results. Converter current measurement at the PCC under the three different unbalance conditions described in Fig. 4. From left to right: Unbalance induced in phase $a$, phase $b$ and phase $c$. From top to bottom: a) phase currents, b) $\alpha, \beta$ components, c), d) and e) are the positive, negative and zero components of the current vector.

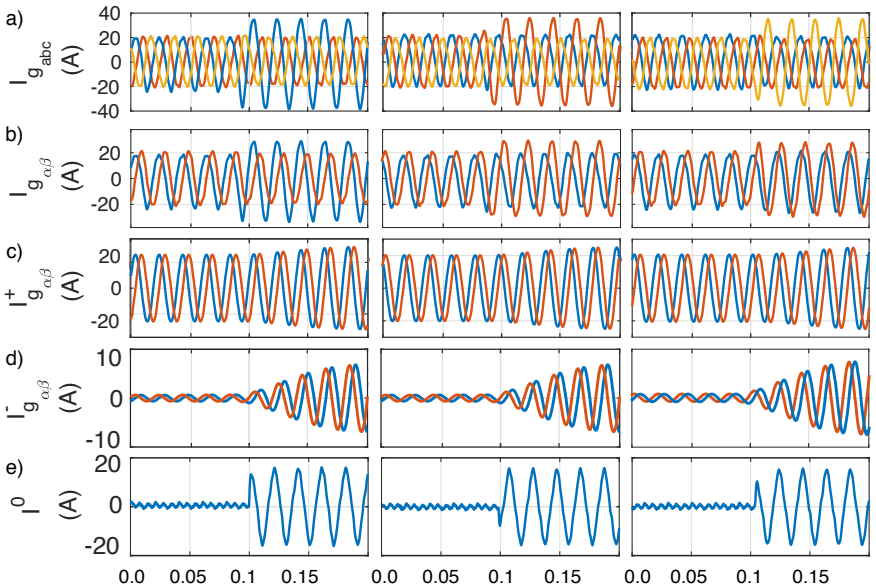

Fig. 6. Experimental results. Grid current measurement, under the three different unbalance conditions described in Fig. 4. From left to right: Unbalance induced in phase $a$, unbalance induced in phase $b$ and unbalance induced in phase $c$. From top to bottom: a) phase currents, b) $\alpha, \beta$ components, c), d) and e) are the positive, negative and zero components of the current vector.

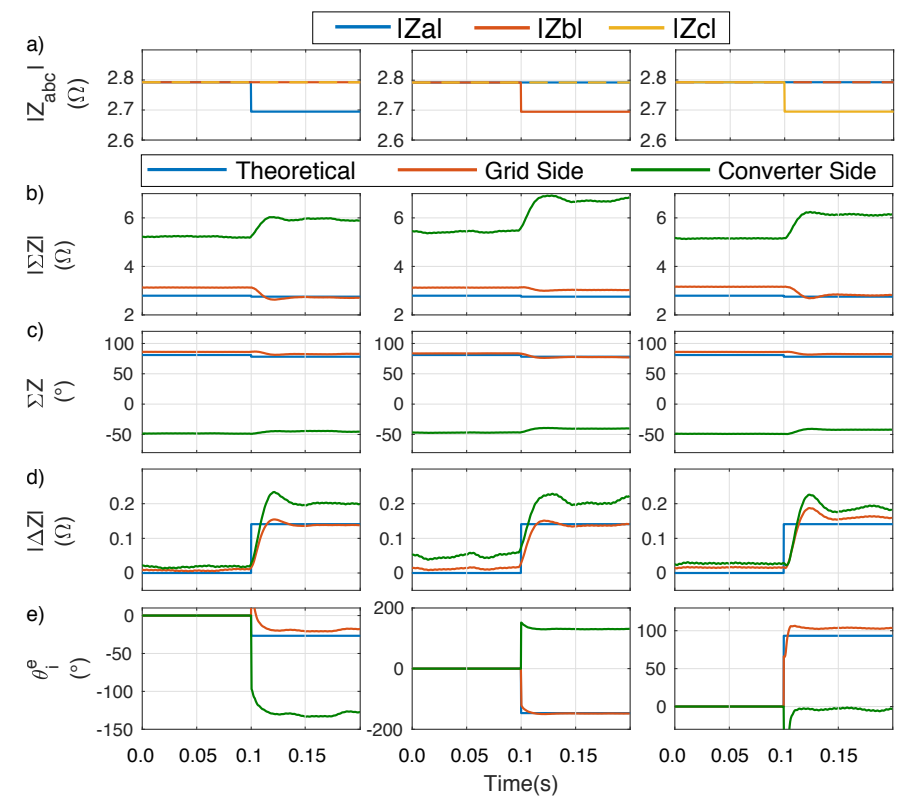

Fig. 7. Experimental Results. Equivalent grid impedance at the PCC measured at $50 \mathrm{~Hz}$. From left to right: Unbalance induced in phase $a$, phase $b$ and phase $c$. From top to bottom: a) phase impedances, b) $|\Sigma Z|$ component, c) phase of $\Sigma Z$, d) $|\Delta Z|$ component and e) $\theta_{i}^{e}$, which corresponds to the unbalance angle.

where $v_{g_{\alpha \beta}}$ and $i_{g_{\alpha \beta}}$ are the PCC voltage and the grid current and $v_{c_{\alpha \beta}}$ and $i_{c_{\alpha \beta}}$ are the voltage and current at the converter side. The required extra sensor for the $i_{g_{\alpha \beta}}$ current is an undesired component, and thus it is desirable to obtain an impedance estimation without it. However, as it is evidenced in Fig. 7, the use of the converter current at the fundamental frequency is not an option. In the following section, an approach for the unbalance estimation based on the injection of a high frequency signal is proposed. The method will enable 
the measurement of the grid impedance from the converter side currents, thus leading to a sensorless compensation.

\section{High-FREQUENCY-SIGNAL INJECTION AND ONLINE MEASUREMENT OF EQUIVALENT IMPEDANCE AT THE PCC}

As discussed previously, the existence of unbalances both in the impedance and in the voltages will generate negative and zero sequence components in the current vector (10), (11). The developed model did not take any assumption about the excitation frequency, and thus it can be used to any fixfrequency excitation just changing the $\omega_{e}$ variable by the frequency of interest. If a balanced high frequency $\left(\omega_{h f}\right)$ carrier-signal voltage, $\mathbf{v}_{\alpha \beta}^{h f+}$, as denoted by (20), is added to the converter voltage command, it will induce a high frequency current (21) that includes a high-frequency negative sequence component determined by the impedance unbalance. However, two fundamental differences exist compared to the fundamental excitation: 1) as the high frequency voltage is created by the converter and superimposed to the fundamental voltage, the resulting high frequency converter current, $\mathbf{i}_{\mathbf{C}}^{h f}$, contains the unbalance information, hence, measuring the current at the load, $\mathbf{i}_{\mathbf{l}}$, or at the grid side, $\mathbf{i}_{\mathrm{g}}$, is not needed to proceed with the compensation (see Fig. 1). Same concern implies the selection of the excitation signal frequency, which should be selected with enough spectral separation from the current controller bandwidth and not higher than half the switching frequency when synchronous sampling is used, according to Nyquist theorem. The selection of an interharmonic is done to not interfere with possible additional shunt-connected converters at the PCC; 2) filtering the negative sequence current from the positive one is easier at higher frequencies, since there is more spectral separation. This will allow to increase the resolution of the system to light unbalanced conditions. Fig. 8 and 9 show, respectively, the experimental high frequency voltage at the PCC and current signals injected by the converter to calculate the equivalent grid impedance. To clarify and ease the understanding the signals are presented in the synchronous reference frame at $333 \mathrm{~Hz}$. At instant $t=0.1 \mathrm{~s}$, the unbalance is produced. As expected, both the negative voltage and current sequences have information about the unbalance.

$$
\begin{gathered}
\mathbf{v}_{\alpha \beta}^{h f}=V^{h f} e^{j \omega_{h f} t} \\
\mathbf{i}_{\alpha \beta}^{h f}=V^{h f} \sum_{i=1}^{n}\left[\Sigma Y_{i}^{h f} e^{j \omega_{h f} t}+\Delta Y_{i}^{h f} e^{j\left(-\omega_{h f} t+\theta_{e}^{i}\right)}\right]
\end{gathered}
$$

The proposed High Frequency Signal (HFS) injection for the estimation of the model parameters has been tested by both simulation and experimental results. The simulation parameters are gathered in Table I. In Fig. 10 the proposed model is verified using Matlab/Simulink ${ }^{\circledR}$ for different steps in the load connected at the PCC.

Fig. 10 gathers the results obtained in the simulation, the theoretical expression and the experimental results of the method. The sequence of the experiment is the same as the one explained above in Fig. 7. A load unbalance is induced in
TABLE II

RMS CURRENT VALUES COMPARISON

\begin{tabular}{c|c|c|c|c|c|c|} 
& \multicolumn{2}{c|}{ Model } & \multicolumn{2}{c|}{ Simulation } & \multicolumn{2}{c}{ Experimental } \\
\cline { 2 - 7 } & \multicolumn{2}{c|}{ (A) } & \multicolumn{2}{c|}{ error $(\%)$} & \multicolumn{2}{c}{ error $(\%)$} \\
\hline$a$ & $t_{1}$ & $t_{2}$ & $t_{1}$ & $t_{2}$ & $t_{1}$ & $t_{2}$ \\
\hline \hline$I_{g_{\alpha \beta}^{+}}^{+}$ & 14.42 & 17.68 & 1.05 & 0.34 & 0.0 & 1.2 \\
$I_{g_{\alpha \beta}}^{-}$ & 0.0149 & 4.288 & 0.0 & 5.2 & 0.0 & 7.87 \\
$I_{\alpha \beta}^{h f^{+}}$ & 0.6632 & 0.7501 & 1.01 & 4.81 & 0.48 & 5.01 \\
$I_{\alpha \beta}^{h f^{-}}$ & 0.0197 & 0.1258 & 0.0 & 3.11 & 0.05 & 3.8 \\
\hline \hline$b$ & $t_{1}$ & $t_{2}$ & $t_{1}$ & $t_{2}$ & $t_{1}$ & $t_{2}$ \\
\hline \hline$I_{g_{\alpha \beta}}^{+}$ & 14.33 & 17.13 & 0.49 & 1.42 & 0.21 & 2.78 \\
$I_{g_{\alpha \beta}}^{-}$ & 0.044 & 4.238 & 0.0 & 3.5 & 0.54 & 7.46 \\
$I_{\alpha \beta}^{h f^{+}}$ & 0.6679 & 0.7442 & 2.5 & 4.9 & 0.83 & 8.95 \\
$I_{\alpha \beta}^{h f^{-}}$ & 0.0196 & 0.125 & 0.0 & 3.01 & 3.16 & 3.73 \\
\hline \hline$c$ & $t_{1}$ & $t_{2}$ & $t_{1}$ & $t_{2}$ & $t_{1}$ & $t_{2}$ \\
\hline \hline$I_{g_{\alpha \beta}}^{+}$ & 14.26 & 17.36 & 1.68 & 1.48 & 0.08 & 3.77 \\
$I_{g_{\alpha \beta}}^{-}$ & 0.0043 & 4.303 & 0.0 & 3.2 & 0.0 & 7.80 \\
$I_{\alpha \beta}^{h f^{+}}$ & 0.6675 & 0.741 & 2.5 & 5.1 & 0.3 & 5.34 \\
$I_{\alpha \beta}^{h f^{-}}$ & 0.0198 & 0.1198 & 0.0 & 3.09 & 1.10 & 8.85 \\
\hline
\end{tabular}

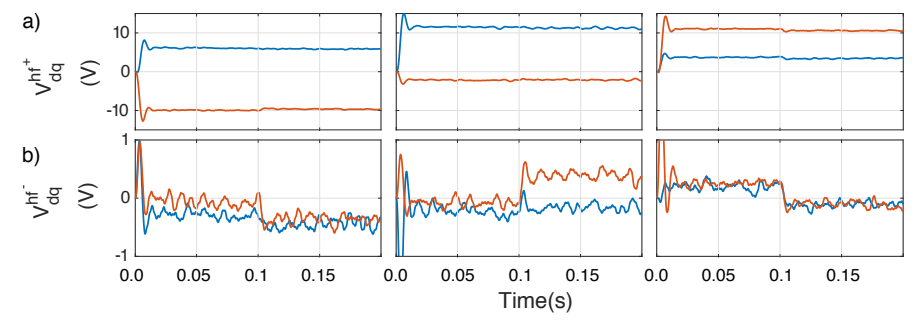

Fig. 8. Experimental results. High frequency PCC voltage. Each column presents the results under one phase unbalanced, left column: unbalance in phase $a$, in the middle: unbalance in phase $b$ and column at the right: unbalance in phase $c$. From top to bottom: a) Positive sequence of the high frequency voltage signal in the $d q$ reference frame at $333 \mathrm{~Hz}, \mathrm{~b}$ ) Negative sequence in the $d q$ reference frame at $333 \mathrm{~Hz}$.

every phase of the system at $0.1 \mathrm{~s}$ and every impedance term is calculated only with the converter side measurements.

Table II summarizes the RMS current values, at $50 \mathrm{~Hz}$ and $333 \mathrm{~Hz}$ ( $h f$ superscript), of the presented model, the simulation and the experimental results. In this table, $t_{1}$ and $t_{2}$, represent the instants before and after the unbalance takes place. Model columns show the current values resulted from applying the mathematical model presented in (10) with same grid impedance values from Table I, before and after the unbalance conditions. Simulation and Experimental columns show the results obtained from a MATLAB/Simulink ${ }^{\circledR}$ model, and from the experimental results respectively. The results are shown, in rows, for each of the phases, and as a percentage error respect to the model results. The current values presented in Table II are calculated based on the equivalent impedance of the system in Fig. 1, both at $50 \mathrm{~Hz}$ and $333 \mathrm{~Hz}$. As it was stated above, grid currents, $I_{g_{\alpha \beta}}^{+}$and $I_{g_{\alpha \beta}}^{-}$, are produced by the interaction of both grid and converter voltages. These estimated grid currents are supported as well as by the results presented in Fig. 6 c) and d). On the contrary, $I_{\alpha \beta}^{h f^{+}}$and $I_{\alpha \beta}^{h f^{-}}$ 


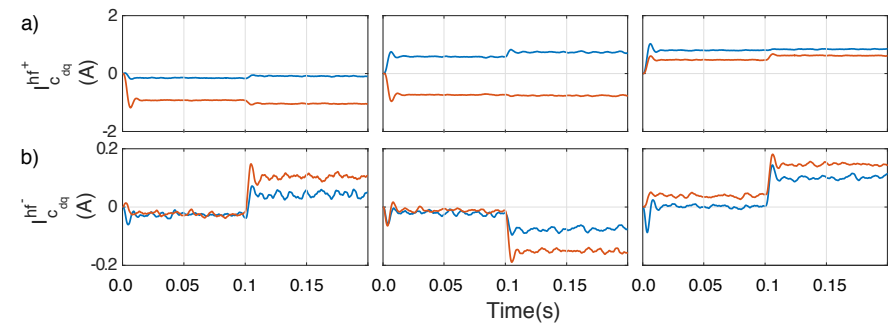

Fig. 9. Experimental results. High frequency converter current. Each column presents the results under one phase unbalanced, left column: unbalance in phase $a$, in the middle: unbalance in phase $b$ and column at the right: unbalance in phase $c$. From top to bottom: a) Positive sequence of the high frequency current signal in the $d q$ reference frame at $333 \mathrm{~Hz}$, b) Negative sequence in the $d q$ reference frame at $333 \mathrm{~Hz}$.

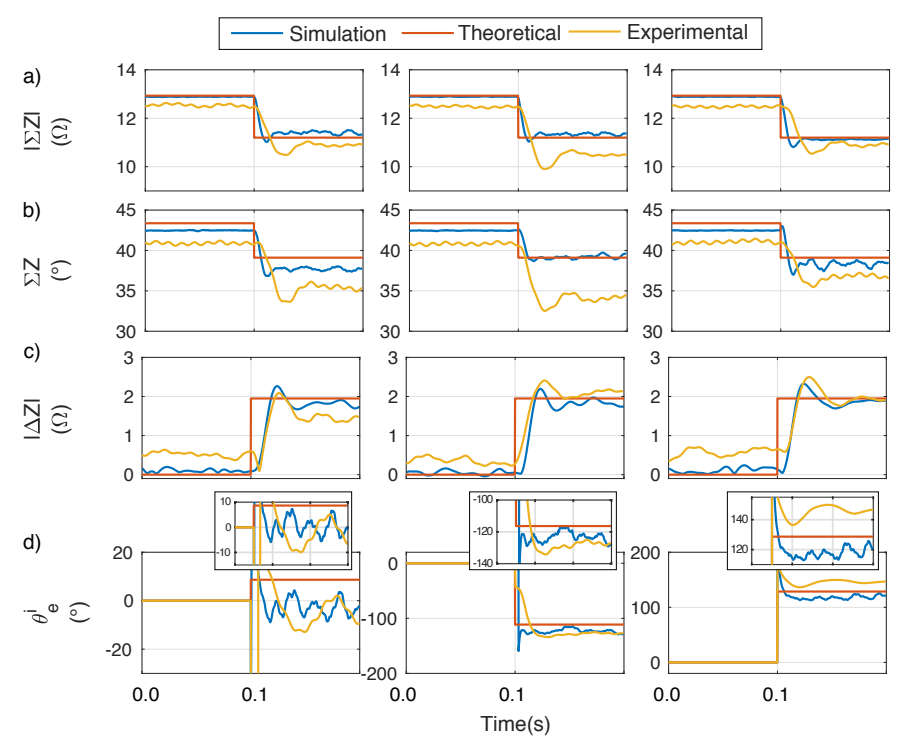

Fig. 10. Comparison of the results obtained in simulation (blue line), theoretical expression (red line) and experimental results (yellow line) of the presented method. Each column presents the results under one phase unbalanced, left column: unbalance in phase $a$, in the middle: unbalance in phase $b$ and column at the right: unbalance in phase $c$. From top to bottom: a) Equivalent value of $\Sigma Z$, b) Angle in deg. of the $\Sigma Z$, c) Equivalent value of $\Delta Z$ and d) $\theta_{e}^{i}$.

result from the high frequency excitation signal (20), provided by the converter, so for this case only the converter voltage at $333 \mathrm{~Hz}$ needs to be considered. Clearly, there is a close agreement among the proposed model, the simulation and the experimental results for all the tested conditions. Nevertheless, small deviations are perceived in the experimental results, due to the fact that the simulations are conducted using an ideal voltage source. This might not be the case in the experimental tests, where a real grid is taken as PCC and thus, include non-ideal voltage conditions. Besides that fact, calibration differences among the three current and voltage sensors are candidates for the error, being below $9 \%$ for all the conducted tests.

\section{HFS-BASED UNBALANCE COMPENSATION}

In order to achieve the proposed sensorless unbalance compensation, it is needed to determine the equivalent differential admittance/impedance at the PCC as seen by the AC network at the fundamental frequency and to relate it with the equivalent high-frequency injected from the power converter. With the definition of variables used in Fig. 1, (10) can be represented in compact form as (22), whereas (21) is given by (23).

$$
\begin{gathered}
I_{g_{\alpha \beta}}^{\omega_{e}}=Y_{e q_{\alpha \beta}}^{\omega_{e}} \cdot\left(V_{g_{\alpha \beta}}^{\omega_{e}}-V_{\alpha \beta}^{\omega_{e}}\right) \\
I_{c_{\alpha \beta}}^{\omega_{h f}}=Y_{e q_{\alpha \beta}}^{\omega_{h f}} \cdot\left(V_{\alpha \beta}^{\omega_{h f}}-V_{g_{\alpha \beta}}^{\omega_{h f}}\right)
\end{gathered}
$$

In (22) and (23), $\omega_{e}$ is the fundamental frequency and $\omega_{h f}$ the high-frequency. The $Y_{e q}$ is the equivalent admittance at the PCC, determined from the parallel connection of the grid and the load impedances as: $Y_{e q}=Y_{g}+Y_{l}$ and can be determined using (13). By analyzing (22) and (23), it is clear that the equivalent admittance can be determined, at the two different frequencies, from both expressions. In traditional unbalance compensation approaches, (22) is used. However, that requires an additional current sensor for the grid or load current. In the method proposed in this paper, only the converter current is measured and thus, the compensation will be determined from (23). It is worth noting that, assuming the grid voltage is represented as an ideal voltage source upstream the grid impedance, the high-frequency component of the grid voltage $\left(V_{g_{\alpha \beta}}^{\omega_{h f}}\right)$ could be assumed to be zero. Compensating the circulating high-frequency negative sequence current, requires to inject a high-frequency negative sequence voltage. Being the goal of the proposed high-frequency injection to determine the needed compensation voltage at the fundamental frequency, the idea is to first determine the needed voltage for the high-frequency excitation, and from there, obtaining the corresponding fundamental frequency component. By applying (10) to the high-frequency components and making the two terms generating a negative sequence current to cancel out, an expression for the required negative sequence high-frequency voltage is given by (24).

$$
\mathbf{v}_{\alpha \beta}^{h f-}=-\mathbf{v}_{\alpha \beta}^{h f+} \sum_{i=1}^{n} \frac{\Delta Y_{i}^{h f} e^{-j \theta_{e}^{i}}}{\Sigma Y_{i}^{h f}}
$$

As the information of the load unbalance is still included in $\mathbf{v}_{\alpha \beta}^{h f-}$, the proper correction action at grid frequency can be derived from this value.

In regard to the high frequency excitation signal, its magnitude and frequency values can be tuned separately. From a power quality perspective, increasing the frequency and reducing the magnitude is preferred. However, this reduces the signal to noise ratio of the method. Additionally, the injected frequency should not be a harmonic of the fundamental frequency, in order to avoid the reaction of any existing harmonic compensator connected to the grid [34]. For this paper, the values shown in Table I have been chosen.

The control scheme shown in Fig. 11 is used to compensate for the negative sequence current at grid frequency. A positive 


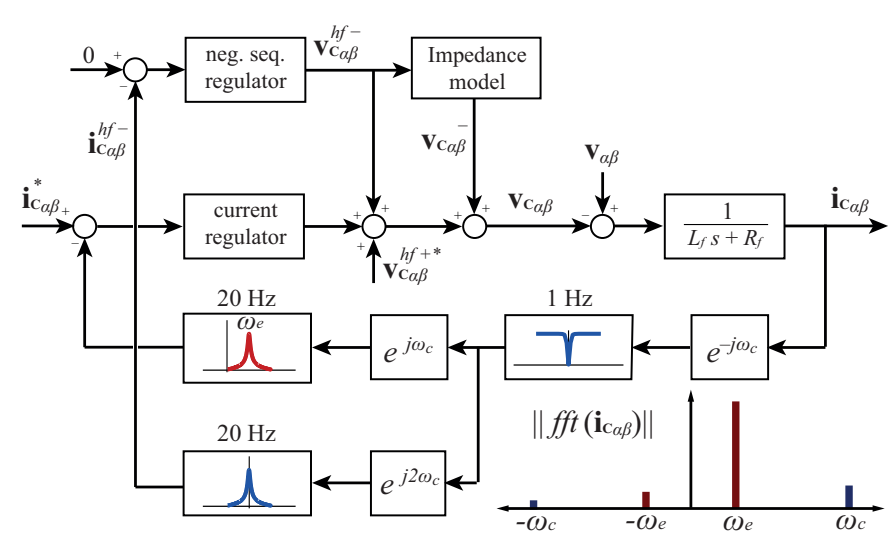

Fig. 11. High frequency signal injection and signal processing. Spectrum showing the main components of the converter current $\left(\mathbf{i}_{\mathbf{c}}\right)$ is shown in the lower right corner.

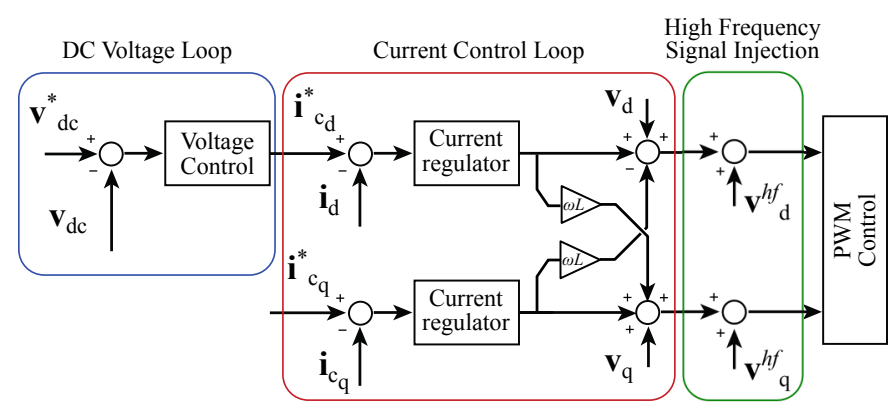

Fig. 12. Conventional cascade control combined with the high frequency excitation signal. High frequency signal is injected as a voltage signal over the current-loop control action.

sequence high frequency $(333 \mathrm{~Hz})$ carrier voltage, $\mathbf{v}_{\mathbf{C} \alpha \beta}^{h f+}$, is added to the converter voltage, causing a negative sequence component to appear in the converter current at the same frequency, $\mathbf{i}_{\mathbf{C} \alpha \beta}^{h f-}$. Provided that the grid side is free from this frequency component and mostly balanced, this negative sequence component is the same that could be observed at the load, i.e. $\mathbf{i}_{\mathbf{C} \alpha \beta}^{h f-}=-\mathbf{i}_{\mathbf{l} \alpha \beta}^{h f-}$. This negative sequence current is obtained from the converter current and controlled to be zero by using two PI regulators, one per each axis. The output of this regulator, $\mathbf{v}_{\mathbf{C} \alpha \beta}^{h f-}$, is used to estimate the negative sequence to be added at grid frequency to the converter voltage, $\mathbf{v}_{\mathbf{C} \alpha \beta}^{-}$, in order to achieve the compensating objective. An unbalance impedance model is used to relate injections at both frequencies, provided that a linear load is connected to the PCC. The unbalance impedance model shown in Fig. 11 which relates the fundamental frequency to the high frequency compensation can be obtained by calculating the differential admittance at the high-frequency corrected by the relationship between the magnitude of the ideal grid voltage (assumed here to be 1 p.u.) and the injected high-frequency voltage. The relationship is given by (25).

$$
v_{c_{\alpha \beta}}^{-}=\Delta Y_{e q}^{h f} \cdot \frac{V_{g}}{V_{c}} \cdot v_{c_{\alpha \beta}}^{h f}
$$

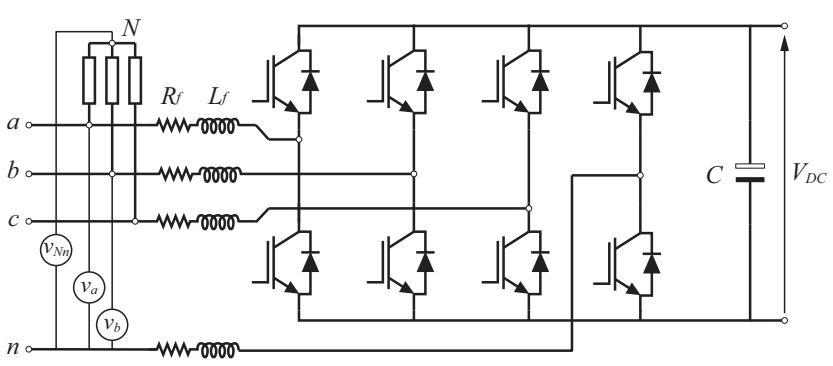

Fig. 13. Zero sequence voltage compensation. Proposed sensor arrangement for the measurement of the zero sequence component.

\section{ZERO-SEQUENCE COMPENSATION}

Zero-sequence currents arise in the LV distribution system as a consequence of both unbalanced and non-linear loads. The local compensation of zero-sequence currents in order to avoid their propagation upstream from the PCC is a desirable ancillary service for power converters, though only four-wire topologies are valid to comply with this function. This task can be easily done by measuring the zero-sequence current at the grid side and implementing a closed loop strategy for compensating it. Unfortunately, this requires not only an additional sensor but also the connection or communication between distant points. A theoretical alternative comes from the compensation of the zero-sequence voltage at the PCC, which could be calculated from the grid phase-voltage sensors of the VSC. However, the use of three voltage sensors ranged at full scale to measure the voltages at the PCC makes it difficult to provide a suitable resolution in the calculation of this value, and it can be even impossible in the interconnection to strong power systems. The arrangement proposed in Fig. 13 for a four-leg topology is used in this paper to solve this adversity. Two full-scale sensors are combined with a third one, ranged at a much lower scale. The latter sensor is devoted to measure the zero-sequence voltage component directly, by connecting it between the grounded neutral point of the installation and the artificial neutral point of a three-phase balanced high impedance load, intentionally located at the PCC. The analytical expression for the zero sequence voltage is given by (26).

$$
v_{N n}=\frac{1}{3}\left(v_{a}+v_{b}+v_{c}\right)=v^{0} .
$$

After applying a second-order low-pass filter to remove the high frequency components of $v_{N_{n}}$, a simple resonant controller could be used to cancel the fundamental frequency zerosequence component seen from the grid side. Nevertheless, a repetitive controller (RC) was selected for this application in order to also remove other zero-sequence frequencies. This is of special interest in this type of installations in which the massive use of single-phase non-linear loads can cause a significant flow of triplen current harmonics. Fig. 14 shows the control loop, which cancels the zero-sequence components by adding the proper signal to the modulation of the neutral leg of the converter. Notice that, although the total modulation of 


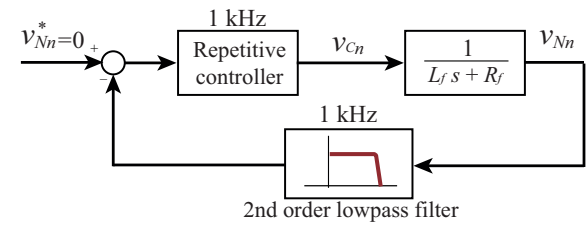

Fig. 14. Zero sequence voltage compensation. Control loop modeling based on the use of a repetitive controller.

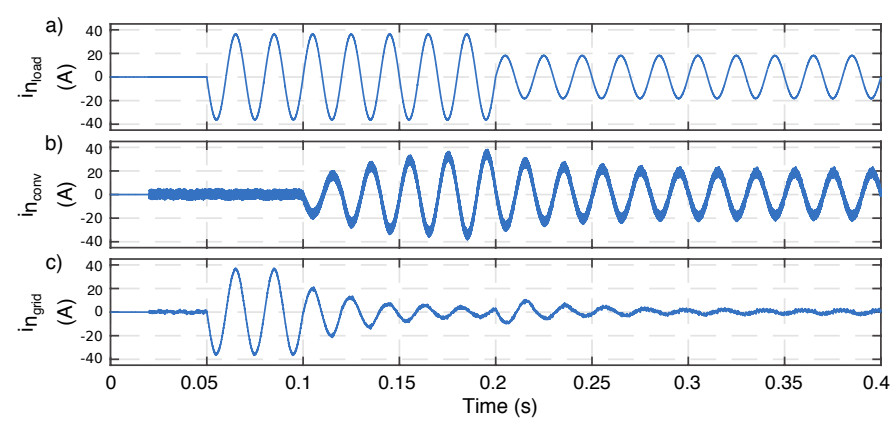

Fig. 15. Simulation results. Zero sequence compensation. (a) neutral current at the load $\left(i_{n_{\text {load }}}\right),(\mathrm{b})$ current at the converter neutral leg $\left(i_{n_{\text {conv }}}\right)$, and (c) neutral current at the grid side $\left(i_{n_{\text {grid }}}\right)$.

this leg contains other zero-sequence components to counteract the effect of the traditional zero sequence injection in the other legs, only the added output of the RC appears in $v_{C n}$. In Fig. 15 , the proper compensation of the zero sequence component is demonstrated. The neutral current at the load $\left(i_{n_{\text {load }}}\right)$ is presented in Fig. 15 a). As it can be seen, two single-phase loads are connected to phase $a$ at time $0.05 \mathrm{~s}$ and one of them is disconnected at time $0.2 \mathrm{~s}$ to test transient performance. The application of the RC proposed in this paper together with the special arrangement of voltage sensors, leads to a fast compensation of grid neutral current $\left(i_{n_{\text {grid }}}\right)$ in Fig. 15 c) by the converter $\left(i_{n_{\text {conv }}}\right)$, which starts the injection at $0.1 \mathrm{~s}$.

\section{CONCLUSions}

In this paper, a method for measuring and compensating unbalance currents for 4-wire 3-phase converters is presented. A high frequency signal injection is used for the negative sequence and a Repetive Controller (RC) for zero sequence components. Both strategies allow a sensorless high-resolution alternative for unbalance compensation. A model considering unbalance loads and voltages has been developed. This model allows to estimate the negative sequence currents generated by the unbalance loads as well as by the cross coupling terms. From the general model, a high frequency model which shows the resulting high frequency current in the presence of unbalance conditions has been obtained.

\section{REFERENCES}

[1] R. Lasseter, "Microgrids," in Power Engineering Society Winter Meeting, 2002. IEEE, vol. 1, 2002, pp. $305-308$ vol.1.

[2] R. Lasseter and P. Paigi, "Microgrid: a conceptual solution," in Power Electronics Specialists Conference, 2004. PESC 04. 2004 IEEE 35th Annual, vol. 6, june 2004, pp. 4285 - 4290 Vol.6.
[3] J. M. Guerrero, "Editorial special issue on power electronics for microgrids; part ii," Power Electronics, IEEE Transactions on, vol. 26, no. 3, pp. $659-663$, march 2011.

[4] N. G. Hingorani and L. Gyugyi, Understanding FACTS: Concepts and Technology of Flexible AC Transmission Systems. New York: IEEE, 2000.

[5] B. Singh, R. Saha, A. Chandra, and K. Al-Haddad, "Static synchronous compensators (statcom): a review," IET Power Electronics, vol. 2, no. 4, pp. 297-324, July 2009.

[6] L. Gyugyi, C. Schauder, and L. Sen, "Static synchronous series compensator: A solid-state approach to the series compensation of transmission lines," IEEE Transactions on Power Delivery, vol. 12, no. 1, pp. 406417, 1997.

[7] V. Khadkikar, "Enhancing electric power quality using upqc: A comprehensive overview," IEEE Transactions on Power Electronics, vol. 27, no. 5, pp. 2284-2297, May 2012.

[8] B. Singh, K. Al-Haddad, and A. Chandra, "A review of active filters for power quality improvement," Industrial Electronics, IEEE Transactions on, vol. 46, no. 5, pp. 960 -971, oct 1999.

[9] V. Corasaniti, M. Barbieri, P. Arnera, and M. Valla, "Comparison of active filters topologies in medium voltage distribution power systems," in Power and Energy Society General Meeting - Conversion and Delivery of Electrical Energy in the 21st Century, 2008 IEEE, july 2008, pp. $1-8$.

[10] H. Fujita and H. Akagi, "The unified power quality conditioner: The integration of series and shunt-active filters," IEEE Transactions on Power Electronics, vol. 13, no. 2, pp. 315-322, 1998.

[11] P. . Cheng and T. . Lee, "Distributed active filter systems (dafss): A new approach to power system harmonics," IEEE Transactions on Industry Applications, vol. 42, no. 5, pp. 1301-1309, Sep. 2006.

[12] F. Bastiao, P. Cruz, and R. Fiteiro, "Impact of distributed generation on distribution networks," in Electricity Market, 2008. EEM 2008. 5th International Conference on European, may 2008, pp. 1 -6.

[13] R. Walling, R. Saint, R. Dugan, J. Burke, and L. Kojovic, "Summary of distributed resources impact on power delivery systems," Power Delivery, IEEE Transactions on, vol. 23, no. 3, pp. 1636 -1644, july 2008.

[14] V. Khadkikar, "Enhancing electric power quality using upqc: A comprehensive overview," IEEE Transactions on Power Electronics, vol. 27, no. 5, pp. 2284-2297, 2012.

[15] M. Savaghebi, A. Jalilian, J. Vasquez, and J. Guerrero, "Autonomous voltage unbalance compensation in an islanded droop-controlled microgrid," IEEE Transactions on Industrial Electronics, vol. PP, no. 99, p. 1, 2012.

[16] C. Po-Tai, C. Chien-An, L. Tzung-Lin, and K. Shen-Yuan, "A cooperative imbalance compensation method for distributed-generation interface converters," IEEE Transactions on Industry Applications, vol. 45, no. 2, pp. 805-815, 2009.

[17] C. J. Melhorn, T. D. Davis, and G. E. Beam, "Voltage sags: their impact on the utility and industrial customers," IEEE Transactions on Industry Applications, vol. 34, no. 3, pp. 549-558, May 1998.

[18] A. Tuladhar, , T. Unger, and K. Mauch, "Control of parallel inverters in distributed ac power systems with consideration of line impedance effect," IEEE Transactions on Industry Applications, vol. 36, no. 1, pp. 131-138, Jan 2000.

[19] American National Standard for Electrical Power Systems and Equipment, Voltage Ratings (60 Hertz). ANSI c84.1-1995.

[20] Voltage characteristics of electricity supplied by public distribution systems. EN50160, 2011.

[21] Electromagnetic compatibility (EMC) Part 4-30:Testing and measurement techniques and Power quality measurement methods. IEC 6100-430, 2003.

[22] J. P. R. A. Méllo, C. B. Jacobina, and M. B. d. Rossiter Corrêa, "Threephase four-wire inverters based on cascaded three-phase converters with four and three legs," IEEE Transactions on Industry Applications, vol. 53, no. 6, pp. 5539-5552, Nov 2017.

[23] M. Bongiorno, J. Svensson, and A. Sannino, "An advanced cascade controller for series-connected vsc for voltage dip mitigation," IEEE Transactions on Industry Applications, vol. 44, no. 1, pp. 187-195, Jan 2008.

[24] V. Khadkikar and A. Chandra, "Upqc-s: A novel concept of simultaneous voltage sag/swell and load reactive power compensations utilizing series inverter of upqc," Power Electronics, IEEE Transactions on, vol. 26, no. 9 , pp. $2414-2425$, sept. 2011. 
[25] V. Khadkikar and A. Chandra, "A novel structure for three-phase fourwire distribution system utilizing unified power quality conditioner (upqc)," IEEE Transactions on Industry Applications, vol. 45, no. 5, pp. 1897-1902, Sep. 2009.

[26] A. Al-Zamil and D. Torrey, "A passive series, active shunt filter for high power applications," Power Electronics, IEEE Transactions on, vol. 16 , no. 1 , pp. $101-109$, jan 2001.

[27] G. A. d. A. Carlos, C. B. Jacobina, and E. C. dos Santos, "Investigation on dynamic voltage restorers with two de links and series converters for three-phase four-wire systems," IEEE Transactions on Industry Applications, vol. 52, no. 2, pp. 1608-1620, March 2016.

[28] A. Luna, J. Rocabert, J. I. Candela, J. R. Hermoso, R. Teodorescu, F. Blaabjerg, and P. Rodríguez, "Grid voltage synchronization for distributed generation systems under grid fault conditions," IEEE Transactions on Industry Applications, vol. 51, no. 4, pp. 3414-3425, July 2015.

[29] A. Suárez-González, P. García, Á. Navarro-Rodríguez, G. Villa, and J. M. Cano, "Sensorless unbalance correction as an ancillary service for lv 4-wire/3-phase power converters," in 2017 IEEE Energy Conversion Congress and Exposition (ECCE), Oct 2017, pp. 4799-4805.

[30] J. C. Vasquez, J. M. Guerrero, M. Savaghebi, J. Eloy-Garcia, and R. Teodorescu, "Modeling, analysis, and design of stationary-referenceframe droop-controlled parallel three-phase voltage source inverters," IEEE Transactions on Industrial Electronics, vol. 60, no. 4, pp. 12711280, April 2013.

[31] G. C. Paap, "Symmetrical components in the time domain and their application to power network calculations," IEEE Transactions on Power Systems, vol. 15, no. 2, pp. 522-528, May 2000.

[32] P. García, M. Sumner, Á. Navarro-Rodríguez, J. M. Guerrero, and J. García, "Observer-based pulsed signal injection for grid impedance estimation in three-phase systems," IEEE Transactions on Industrial Electronics, vol. 65, no. 10, pp. 7888-7899, Oct 2018.

[33] M. Saad, H. Ali, H. Liu, S. Khan, H. Zaman, B. M. Khan, D. Kai, and J. Yongfeng, "A dq-domain impedance measurement methodology for three-phase converters in distributed energy systems," Energies, vol. 11, no. 10, 2018. [Online]. Available: http://www.mdpi.com/1996$1073 / 11 / 10 / 2732$

[34] F. Briz, P. García, M. W. Degner, D. Díaz-Reigosa, and J. M. Guerrero, "Dynamic behavior of current controllers for selective harmonic compensation in three-phase active power filters," IEEE Transactions on Industry Applications, vol. 49, no. 3, pp. 1411-1420, May 2013. 